\title{
Do We Need Dark Energy to Explain the Cosmological Acceleration?
}

\author{
Felix M. Lev \\ Artwork Conversion Software Inc., Manhattan Beach, USA \\ Email: felixlev314@gmail.com \\ Received June 28, 2012; revised July 30, 2012; accepted August 7, 2012
}

\begin{abstract}
The phenomenon of the cosmological acceleration discovered in 1998 is usually explained as a manifestation of a hypothetical field called dark energy which is believed to contain more than $70 \%$ of the energy of the Universe. This explanation is based on the assumption that empty space-time background should be flat and hence a nonzero curvature of the background is a manifestation of a hidden matter. We argue that quantum theory should proceed not from spacetime background but from a symmetry algebra. Then the cosmological acceleration can be easily and naturally explained from first principles of quantum theory without involving empty space-time background, dark energy and other artificial notions. We do not assume that the reader is an expert in the given field and the content of the paper can be understood by a wide audience of physicists.
\end{abstract}

Keywords: Quantum Theory; De Sitter Invariance; Cosmological Constant

\section{Introduction}

The discovery of the cosmological acceleration (see e.g. Refs. [1,2]) has ignited a vast discussion on how this phenomenon should be interpreted. The results of the observations are usually represented in terms of the parameter which is called the cosmological constant (CC) and denoted by $\Lambda$. The meaning of this quantity will be discussed below. According to Refs. [3,4], the observational data on the value of $\Lambda$ define it with the accuracy better than $5 \%$. Therefore the possibilities that $\Lambda=0$ or $\Lambda<0$ are practically excluded.

The fact that $\Lambda>0$ is usually explained as a manifestation of a hypothetical field called dark energy. The explanation has its roots in the well known debate between Einstein and de Sitter and one of the problems in the debate was whether the curved empty space-time background has a physical meaning or not. This problem is discussed in a vast literature and it encounters serious difficulties known as the $\mathrm{CC}$ problem or dark energy problem. The arguments leading to dark energy are discussed in detail in Section 2. On the other hand, in Section 3 we argue that quantum theory should start not from the choice of the space-time background but from the choice of a symmetry algebra. Then the cosmological acceleration can be easily and naturally explained from first principles of quantum theory without involving empty space-time background, dark energy and other artificial notions.
We do not assume that the reader is familiar with the Einstein equations and de Sitter symmetry. We tried to make the presentation of the material as simple as possible and we believe that the content of the paper can be understood by a wide audience of physicists.

\section{Arguments Leading to Dark Energy}

The majority of works dealing with the CC problem proceed from the assumption that the gravitational constant $G$ is the fundamental physical quantity, the goal of the theory is to express $\Lambda$ in terms of $G$ and to explain why $\Lambda$ is so small. For this reason we first discuss whether indeed $G$ can be treated as a fundamental constant and whether the theory should explain the value of $\Lambda$.

The quantity $G$ defines the gravitational force in the Newton law of gravity. Numerous experimental data show that this law works with a very high accuracy. However, this only means that $G$ is a good phenomenological parameter. At the level of the Newton law one cannot prove that $G$ is the exact constant which does not change with time, does not depend on masses, distances etc.

General Relativity (GR) is a classical (i.e. non-quantum) theory based on the minimum action principle. We will not assume that the reader is familiar with the Einstein equations. The only features of these equations which are important for our discussion are the following. The left-hand-side of these equations contain quantities 
describing the properties of space-time - the Ricci tensor $R_{\mu v}$, the metric tensor $g_{\mu v}$ and the tensor of the scalar curvature $R_{c}(\mu, v=0,1,2,3)$, while the right-hand-side contains stress energy tensor of matter $T_{\mu v}$. The Einstein equations derived from the minimum action principle read

$$
R_{\mu v}-\frac{1}{2} g_{\mu v} R_{c}=\left(8 \pi G / c^{4}\right) T_{\mu v}
$$

Therefore $G$ is the coefficient of proportionality between the left-hand-side and rihgt-hand-side. General Relativity cannot calculate $G$ or give a theoretical explanation why this value should be as it is. A problem arises whether the quantity $G$ should be treated as a fundamental or phenomenological constant. For example, the quantity $c$ is usually treated as fundamental and then the problem of calculating $c$ does not arise. One can say that the value of $c$ is as it is simply because we wish to measure time in seconds and distances in meters. One might think that the quantity $G$ can be treated analogously and its value is as it is simply because we wish to measure masses in kilograms and distances in meters (in the spirit of Planck units). However, treating $G$ as a fundamental constant can be justified only if there are strong reasons to believe that the Lagrangian of GR is the only possible Lagrangian. Let us consider whether this is the case.

The Lagrangian of GR should be invariant under general coordinate transformations and the simplest way to satisfy this requirement is a choice when it is proportional to $R_{c}$. In this case the Newton gravitational law is recovered in the nonrelativistic approximation and the theory is successful in explaining several well-known phenomena. However, the argument that this choice is simple and agrees with the data, cannot be treated as a fundamental requirement. Another reason for choosing the linear case is that here equations of motions are of the second order while in quadratic, cubic cases etc. they will be of higher orders. However, this reason also cannot be treated as fundamental. It has been argued in the literature that GR is a low energy approximation of a theory where equations of motion contain higher order derivatives. In particular, a rather popular approach is when the Lagrangian contains a function $f\left(R_{c}\right)$ which should be defined from additional considerations. In that case the constant $G$ in the Lagrangian is not the same as the standard gravitational constant. It is believed that the nature of gravity will be understood in the future quantum theory of gravity but efforts to construct this theory has not been successful yet. Hence there are no solid reasons to treat $G$ as a fundamental constant.

Special Relativity works with Minkowski space, which is also called the space of events. It is very important to note that Minkowski space has a physical meaning only as a space of events for real bodies. In particular, the notion of empty space has no physical meaning since it contradicts the physical principle that a definition of a physical quantity is a description of how this quantity should be measured. In particular, one can discuss how coordinates of real bodies can be measured but there is no way to measure coordinates of the empty space which exists only in our imagination.

Physicists consider others spaces of events, for example de Sitter (dS) space. It is a set of points characterized by five coordinates $(t, x, y, z, u)$ which satisfy the restriction $x^{2}+y^{2}+z^{2}+u^{2}-t^{2}=R^{2}$ where $R$ is some parameter, we work in units where $c=1$ and hence time $t$ has the same dimension as the spacial coordinates $(x, y, z, u)$. The dS space is invariant under the action of the dS group, which contains only conventional and hyperbolic rotations. Therefore the action of the dS group on $\mathrm{dS}$ space does not depend on $R$ at all and, instead of the quantities $(t, x, y, z, u)$ satisfying the above restriction, one can characterize points on the dS space by dimensionless quantities

$$
\left(\xi_{0}=t / R, \xi_{1}=x / R, \xi_{2}=y / R, \xi_{3}=z / R, \xi_{4}=u / R\right)
$$

satisfying the restriction $\xi_{1}^{2}+\xi_{2}^{2}+\xi_{3}^{2}+\xi_{4}^{2}-\xi_{0}^{2}=1$.

An analogy of this situation follows. Suppose that a one-dimensional man lives on a circumference in the $\mathrm{xy}$ plane with the center in the origin and radius $R$. The man does not know that in the two-dimensional world the circumference is described by the coordinates $(x, y)$ satisfying $x^{2}+y^{2}=R^{2}$ since he has no information about $x, y$ and $R$. However he can measure distances and describe the geometry of his one-dimensional world in terms of a dimensionless parameter $\varphi \in[0,2 \pi]$.

Consider a vicinity of the North pole of $\mathrm{dS}$ space assuming that the pole has the coordinates $(0,0,0,0, R)$. If we consider only such points of dS space that $u$ is close to $R$ and all the values of $(t, x, y, z)$ are much less than $R$ then in this vicinity, geometry is very close to that of Minkowski space. The dimension of the quantities $(t, x, y, z)$ in this vicinity depends on the dimension in which $R$ is measured. The curvature of dS space in terms of $(t, x, y, z)$ is $\Lambda=3 / R^{2}$. Then the experimental results [1-3] say that $R$ is of order $10^{26} \mathrm{~m}$. This discussion shows that in $\mathrm{dS}$ theory $\Lambda$ is not present at all; it appears only when one wishes to parametrize dS space by dimensionful coordinates. Hence the question of why $\Lambda$ is as it is, is not fundamental since the answer is: because we want to measure distances in meters. In particular, there is no guaranty that $\Lambda$ will not change with time.

When the Lagrangian is linear in $R_{c}$, the most general Einstein equations are not (1) but

$$
R_{\mu v}-\frac{1}{2} g_{\mu \nu} R_{c}+\Lambda g_{\mu v}=\left(8 \pi G / c^{4}\right) T_{\mu \nu}
$$

As follows from this expression, in GR the curvature 
and the metric depend on the presence of matter. In the formal limit, when matter disappears, solutions of Equation (2) are Minkowski space when $\Lambda=0$, dS space when $\Lambda>0$ and anti-de Sitter (AdS) space when $\Lambda<0$. In this connection the following extremely important question arises. As discussed above, these spaces have a physical meaning only as spaces of events for real bodies. At the same time, in GR those spaces arise as solutions of the Einstein equations when matter is absent. In other words, those spaces arise only as empty spaces. Of course, in mathematics one can consider different spaces without thinking about the physical meaning of empty space. But in physics the notion of empty space has no meaning. We believe that these remarks show that the formal limit of GR when matter disappears is unphysical.

In textbooks on gravity written before 1998 (when the cosmological acceleration was discovered) it is often claimed that $\Lambda$ is not needed since its presence contradicts the philosophy of GR: matter creates curvature of space-time, so in the absence of matter space-time should be flat (i.e. Minkowski) while empty dS space is not flat. As noted above, such a philosophy has no physical meaning since the notion of empty space is unphysical. That's why the discovery of the fact that $\Lambda \neq 0$ has ignited many discussions. The most popular approach is as follows. One can move the term with $\Lambda$ in Equation (2) from the left-hand side to the right-hand one:

$$
R_{\mu v}-\frac{1}{2} g_{\mu v} R_{c}=\left(8 \pi G / c^{4}\right) T_{\mu v}-\Lambda g_{\mu v}
$$

Then the term with $\Lambda$ is treated as the stress-energy tensor of a hidden matter which is called dark energy: $\left(8 \pi G / c^{4}\right) T_{\mu \nu}^{D E}=-\Lambda g_{\mu \nu}$. With the observed value of $\Lambda$ this dark energy contains approximately $75 \%$ of the energy of the Universe. In this approach $G$ is treated as a fundamental constant and one might try to express $\Lambda$ in terms of $G$. The existing quantum theory of gravity cannot perform this calculation unambiguously since the theory contains strong divergences. With a reasonable cutoff parameter, the result for $\Lambda$ is such that in units where $\hbar=c=1, G \Lambda$ is of order unity. This result is expected from dimensionful considerations since in these units, the dimension of $G$ is length $^{2}$ while the dimension of $\Lambda$ is $1 /$ length $^{2}$. However, this value of $\Lambda$ is greater than the observed one by 122 orders of magnitude. This problem is called the CC problem or dark energy problem.

Several authors criticized this approach from the following considerations. GR without the contribution of $\Lambda$ has been confirmed with a good accuracy in experiments in the Solar System. If $\Lambda$ is as small as it has been observed then it can have a significant effect only at cosmological distances while for experiments in the Solar System the role of such a small value is negligible. The authors of Ref. [5] titled "Why All These Prejudices Against a Constant?", note that even in a special case $f\left(R_{c}\right)=R_{c}$, the most general form of the Einstein equations is as in Equation (2) and so it is not clear why we should think that only a special case (1) is allowed. If we accept the theory containing a phenomenological constant $G$ which is taken from the outside then why can't we accept a theory containing two independent phenomenological constants?

It is also well known since the 1930s that on quantum level space-time coordinates are not measurable (see e.g. Ref. [6]). Hence on quantum level space-time cannot be described by differential geometry. There exist many papers the authors of which propose their solutions of the $\mathrm{CC}$ problem. In the next section we give simple arguments showing that the $\mathrm{CC}$ problem does not exist and the cosmological acceleration can be easily and naturally explained from first principles of quantum theory.

\section{Quantum Approach to Cosmological Acceleration}

The usual approach to dS symmetry on quantum level is as follows. Since classical dS space is invariant under the action of the dS group, in dS quantum theory operators of dS angular momenta

$$
M^{a b}\left(a, b=0,1,2,3,4 ; M^{a b}=-M^{b a}\right)
$$

should satisfy the commutation relations of the $\mathrm{dS}$ algebra

$$
\begin{aligned}
& {\left[M^{a b}, M^{c d}\right]} \\
& =-i\left(\eta^{a c} M^{b d}+\eta^{b d} M^{a c}-\eta^{a d} M^{b c}-\eta^{b c} M^{a d}\right)
\end{aligned}
$$

where $\eta^{a b}$ is the diagonal metric tensor such that $\eta^{00}=-\eta^{11}=-\eta^{22}=-\eta^{33}=-\eta^{44}=1$. This approach is in the spirit of the well-known Klein's Erlangen program in mathematics.

However, as we argue in Refs. [7,8], quantum theory should not be based on classical space-time background and the approach should be the opposite. Each system is described by a set of independent operators. By definition, the rules how these operators commute with each other define the symmetry algebra. In particular, by definition, dS symmetry on quantum level means that the operators commute according to Equation (4). In semiclassical approximation, quantum theory can recover results obtained by classical one with dS space (see below). In that case $\mathrm{dS}$ space is meaningful only as a space of events for real particles but not as an empty space-time background.

The anti-de Sitter (AdS) symmetry on quantum level can be defined analogously but the value of $\eta^{44}$ in Equation (4) is 1 instead of -1 . Poincare symmetry is a 
special case of dS or AdS symmetry obtained as follows. If $R$ is a parameter with the dimension length and the energy-momentum operator $P^{\mu}$ is defiend as $P^{\mu}=M^{4 \mu} / R$ then in the formal limit $R \rightarrow \infty$ one gets commutation relations of the Poincare algebra from Equation (4). It is clear that on quantum level dS and AdS theories can be constructed without parameters having the dimension of length. Such parameters may be used if one wishes to interpret the results in classical approximation or in Poincare limit but they are not fundamental. In particular, neither $\Lambda$ nor $G$ can be fundamental in agreement with the discussion in the preceding section.

The next step in our construction is the definition of elementary particle. Although theory of elementary particles exists for a rather long period of time, there is no commonly accepted definition of elementary particle in this theory. In Refs. $[7,8]$ we argue that, in the spirit of Wigner's approach to Poincare symmetry, a general definition, not depending on the choice of the classical background and on whether we consider a local or nonlocal theory, is that a particle is elementary if the set of its wave functions is the space of an irreducible representation (IR) of the symmetry algebra in the given theory. The meaning of IR is that the linear space of all possible wave functions cannot be decomposed into a sum of spaces where the operators act independently. Hence the term "irreducible" can be treated as a mathematical synonym of "elementary". There exists a wide literature describing how IRs of the dS, AdS and Poincare algebra can be constructed. Such a construction can be used not only for describing elementary particles but even for describing the motion of a macroscopic body as a whole. For example, when we consider a system of two macroscopic bodies such that the distance between them is much greater than their sizes, it suffices to describe each body as a whole by using the IR with the corresponding mass.

The following important remarks are in order. In Quantum Field Theory, Lagrangians and Minkowski space play only an auxiliary role for constructing the operators $\left(P^{\mu}, M^{\mu v}\right)$ for systems of interacting fields. Hence if we consider only systems of free particles, neither Lagrangian nor Minkowski space is needed. Analogous remarks are valid in dS theory. In particular, for describing systems of free particles, neither Lagrangian nor dS space is needed.

The above notions are sufficient for describing systems of free particles in Poincare, dS and AdS quantum theories. In particular, in semiclassical approximation one can calculate the relative acceleration of two free particles in such theories. One might think that since the particles are free, their relative acceleration will be zero. This is true in Poincare invariant theory but in the $\mathrm{dS}$ and AdS cases the relative acceleration is not zero. The cal- culation of the relative acceleration involves the following steps.

At the starting point we have no space-time and no dimensionful parameters. The only information we have is how wave functions describing particles under consideration are constructed and how the operators $M^{a b}$ act on such wave functions. This is the maximum possible information in quantum theory.

The next step is that we introduce a parameter $R$ with the dimension length and instead of the dS operators $M^{4 \mu}$ work with the energy operator $E=M^{40} / R$ and the momentum operator $P$ such that $P^{k}=M^{4 k} / R$

$(k=1,2,3)$. Then we define classical time $t$ as a parameter describing the evolution according to the Schroedinger equation and define the position operator $\mathbf{r}_{j}$ of particle $j(j=1,2)$ such that it acts on wave functions $\psi\left(\mathbf{p}_{j}\right)$ of particle $j$ in momentum representation as $i \hbar \partial / \partial \mathbf{p}_{j} \quad$ (as in standard quantum mechanics).

A standard quantum-mechanical calculation, which is described in detail in Refs. [7,9-11] (where we discussed different properties of dS quantum theory), shows that in the dS case the classical relative acceleration a of two free particles is $\mathbf{a}=\Lambda c^{2} \mathbf{r} / 3$ where $\mathbf{r}$ is the classical vector of the relative distance between the particles and $\Lambda=3 / R^{2}$. From the formal point of view, the result is the same as in GR on dS space. However, our result has been obtained by using only standard quantum-mechanical notions while dS space, its metric, connection etc. have not been involved at all. The derivation clearly demonstrates that $R$ is not a fundamental quantity but simply a parameter defining the scale of classical spacetime coordinates $(\mathbf{r}, t)$ (in agreement with the remarks in the preceding section).

\section{Conclusion}

In Section 2 we argue that neither $G$ nor $\Lambda$ can be fundamental and the notion of empty space-time background is not physical. Hence the discussion on whether the empty space-time background can be curved or not does not have a physical meaning. In Section 3 we argue that quantum theory should start not from the choice of the empty space-time background but from the choice of a symmetry algebra. In view of this approach, space-time coordinates have a physical meaning only on classical level when they are applied for describing real bodies but not for describing the empty space-time background. In this approach the data of Refs. [1-3] that $\Lambda>0$, should be interpreted not such that the space-time background is dS space but that the dS algebra is more pertinent than the Poincare or AdS ones. As shown in our Ref. [8] and references therein, this opens a radically new approach to gravity where the quantity $G$ is not taken from the outside but (in principle) can be calculated. The above dis- 
cussion shows that the phenomenon of cosmological acceleration can be easily and naturally explained from first principles of quantum theory without involving spacetime background, dark energy and other artificial notions.

\section{Acknowledgements}

The author is grateful to Volodya Netchitailo for reading the manuscript and important remarks and to the anonymous referee for important suggestions on improving the manuscript.

\section{REFERENCES}

[1] S. Perlmutter, G. Aldering, G. Goldhaber, et al., "Measurement of Omega and Lambda from H42 High-Redshift Supernovae," Astrophysical Journal, Vol. 517, No. 2, 1999, pp. 565-586. doi:10.1086/307221

[2] A. Melchiorri, P. A. R. Ade, P. de Bernardis, et al., "A Measurement of Omega from the North American Rest Flight of Boomerang," Astrophysical Journal, Vol. 536, No. 2, 2000, pp. L63-L66. doi:10.1086/312744

[3] D. N. Spergel, R. Bean, O. Dore, et al., "Wilkinson Microwave Anisotropy Probe (WMAP) Three Year Results: Implications for Cosmology," Astrophysical Journal, Vol.
170, No. 2, 2007, pp. 377-408. doi:10.1086/513700

[4] K. Nakamura and Particle Data Group, "Review of Particle Physics," Journal of Physics, Vol. G37, No. 7A, 2010, Article ID: 075021.

[5] E. Bianchi and C. Rovelli, "Why All These Prejudices against a Constant?" 2010, arXiv:1002.3966v3

[6] T. D. Newton and E. P. Wigner, "Localized States for Elementary Systems," Review of Modern Physics, Vol. 21, No. 3, 1949, pp. 400-405. doi:10.1103/RevModPhys.21.400

[7] F. Lev, "Positive Cosmological Constant and Quantum Theory," Symmetry, Special Issue: Quantum Symmetry, Vol. 2, No. 4, 2010, pp. 1945-1980.

[8] F. Lev, "De Sitter Symmetry and Quantum Theory," Physical Review, Vol. D85, No. 3, 2012, Article ID: 065003.

[9] F. Lev, "Finiteness of Physics and Its Possible Consequences," Journal of Mathematical Physics, Vol. 34, No. 2, 1993, pp. 490-527. doi:10.1063/1.530257

[10] F. Lev, "The Problem of Interactions in de Sitter Invariant Theories," Journal of Physics, Vol. A32, No. 7, 1999, pp. 1225-1240.

[11] F. Lev, "Could Only Fermions Be Elementary?" Journal of Physics, Vol. A37, No. 9, 2004, pp. 3287-3304. 